\section{Histopathology of the Skin}

Walter J. Lever, M.D. Third edition. Pp. vii + 653 with 320 illustrations. London: Pitman Medical. Philadelphia: J. B. Myincott. 1961. £6.

It seems almost presumptuous for a dermatologist to review the new edition of Lever's book, so used is one to its being an authoritative guide in the histopathology of the skin. Here is Lever in an up-to-date, more valuable form, preserving intact the virtues of the earlier editions.

The general classification used by the author is under the headings of congenital, non-infectious vesicular and bullous diseases, non-infectious inflammatory diseases, drug eruptions, degenerative diseases, diseases caused by bacteria, fungi, protozoa and viruses, metabolic diseases, tumours and the lymphoma and myelosis group. It is, therefore, easy to follow and explore.

In this third edition, seven years after the second, much has been rewritten. Emphasis is laid on the developing techniques of histochemistry and electron microscopy. Several newly recognized entities of great interest to dermatologists-e.g. sub-corneal pustulosis and kerato-acanthoma-have now been assimilated into the book. A few diseases previously recognized but not discussed by Lever have now been included, e.g. lethal midline granuloma of the face.

There is, as before, an abundance of good black and white photographs, but the text is perhaps more important, providing a clear dissertation on the histological features of every feature under discussion, with brief notes on the clinical appearance; the attention paid to differential (histological) diagnosis is especially valuable. Unobtrusively the reader is given an account in which fact and theory, experimental and clinical findings are given their due share. The style is of consistent clarity.

There are excellent introductory chapters on techniques, embryology and the histology of normal skin. The book is rounded off by a useful glossary and a good index. One of the greatest merits of this book is the provision of reasonably full and up-to-date lists of references following each chapter; lists which are not narrowly confined to American sources but which draw upon the dermatological literature of the world.

This book, the new edition no less than the old, is indispensable for the dermatologist and for any pathologist dealing with dermatological material.

\section{An Atlas of Histopathology of the Skin}

G. H. Percival, G. L. Montgomery and T. C. Dopds. Second edition. Pp. xi + 491. 525 photographs in colour. Edinburgh and London: E. \& S. Livingstone. 1962. £6 ros.

This book is essentially an Atlas. With this second edition, five years after the first, a text has been written to link the photographs, some contents have been included which were not in the first edition. and there are some new illustrations.

It is well produced and the colour is undoubtedly a help for the student of histology, especially when looked at as the authors advocate in artificial (filament lamp) light.

There is a short guide to terminology and a good index. In the reviewer's opinion, however, the text suffers from the lack of a bibliography and from its classification of the subject matter; the authors say $\because$. the subject has been arranged in groups according to the component part of the skin which shows the most structural alteration ... the salient histological changes which occur in the tissues in each group can be easily contrasted and this would seem to compensate for the fact that conditions which have no etiological relationship are to be found in close proximity'. Scabies under this scheme is grouped under diseases affecting the epidermis, and is given only a cross reference in the section on diseases caused by animal parasites. How ever, this classification has clearly been given much thought by the authors, and any method has its dra backs; here the good index mitigates any inconvenience.

In their brief linking text the authors contrive well give a brief account of morphology and histology withot straying into clinical uncertainties. Many dermatologis however, would consider unsatisfactory an account mycosis fungoids not mentioning by name the Pautrier micro-abscesses, of rosacea-like tuberculide under the heading of sarcoidosis and without further explanation of its already misleading name, of bullous diseases witb the only mention of pemphigoid a casual one under the heading of dermatitis herpetiformis. Some new and now fairly well recognized entities are included-e.g? alopecia mucinosa and elastoma perforans verruciforn -but others well established are not-e.g. granular celled myoblastoma and Jessner's lymphocytic infiltration of the skin.

Though not comprehensive or authoritative this book is valuable for the excellent illustrations, and would tef best used as an introduction to the subject or in cons. junction with a reliable textbook of dermatology.

Fingerprint, Palms and Soles; An Introduction to Dermatoglyphics

H. Cummins, Ph.D., and C. Midlo, m.D. Pp. xi 319, illustrated. New York: Dover Books. I $96 \$$ $\gg 1.95$.

One's first impression on reading this book is surprise that so much can be written on the subject-yetothe preface says this is an introduction to the subjes dermatoglyphics and that it does not aim to be a prines to those engaged in its chief practical application, i.e. criminology.

There is, none the less a large bibliography for the reader wishing to go into these aspects more thoroughl $\overline{8}$.

The work is divided into three parts. The first deads with the historical background, and much of this $\frac{\mathcal{O}}{\Phi s}$ fascinating, though presented somewhat diffusely. The possible significance for instance of fingerprints pottery is discussed and that of design suggestim fingerprints in burial chambers. The development $\theta$ the scientific aspects is dealt with in detail.

Part II deals extensively with the minutiæ of methods description and classification.

Part III deals with 'the more fundamental biologic phases of dermatoglyphics'. This surveys comparatife work-humans and other primates-, racial status, work in embryology, genetics and in possible links wigh various diseases.

There is a supplement consisting of anecdotes on the identification of criminals and exoneration of the innocent which seems curiously out of keeping with the rest of the book. The index is very good.

The book is written in American, which is a language in its own right and should not be judged by standaras applied to English; but the American here is both turgid and diffuse, and the style in general leaves mud to be desired-e.g. "The student of dermatoglyphides will not fall into the error of presuming that dermato glyphic features now provide ready answers for re fractory questions in the history of races, particulaty when he sees that attacks from many quarters have faile to yield a completely knit scheme of the affinity races'.

Its main value to the general medical reader wout appear to be the revelation of a great body of wor 